\title{
La prisión del ser: The Rise of Life on Earth de Joyce Carol Oates
}

\author{
Marina FE \\ Universidad Nacional Autónoma de México
}

\begin{abstract}
There beneath her heart there in that small sac of a space where a baby might grow, where the medical books in her father's library showed that a baby might grow, she lived in secret from them. From all of them.

Joyce Carol Oates, Wonderland
\end{abstract}

Una mujer encerrada en su cuerpo, con una vida secreta. Una mujer fea, en un cuerpo que le resulta ajeno y al mismo tiempo irrenunciable, como una prisión perpetua. Un ser precario, marcado por la diferencia, deficiente a los ojos de los demás, de los otros. Así son muchos de los personajes femeninos en las novelas y los cuentos de la escritora neoyorkina Joyce Carol Oates, personajes como la protagonista de The Rise of Life on Earth: ${ }^{1}$

Kathleen Hennessy with her pie-shaped face, pie-shaped maturing breasts, her pale, plump, soft, seemingly textureless flesh like that of a mollusk pried from its shell... and her recessed eyes that were darkly bright and alert, though betraying no expression; her delicate complexion riddled with tiny pimples like buckshot [...] and something precocious about her small, pert, moist, pink rosebud of a mouth, a miniature mouth, that reminded observers of a part of the female anatomy that is private and should not be exposed to casual eyes (3-4).

En la narrativa de Joyce Carol Oates descubrimos una sociedad decadente, desordenada y anómica, donde los individuos, particularmente las mujeres, parecen estar totalmente desorientados o perdidos. Se trata de la sociedad norteamericana contemporánea, desgastada por la violencia hacia

' Joyce Carol OATES, The Rise of Life on Earth. Las paginas correspondientes aparecerán entre paréntesis después de cada cita. 
afuera y, sobre todo, hacia adentro, hacia la propia comunidad paralizada por su incapacidad por cambiar las cosas.

En muchos de sus cuentos y novelas aparecen personajes femeninos que, de una u otra manera, resultan ser víctimas de esta sociedad y que personifican el problema de la identidad femenina, así como la relación conflictiva de las mujeres con su propio cuerpo. En su nuevo país de las maravillas (o de las pesadillas), su "Wonderland" americano, las mujeres tienen, desde la infancia y la adolescencia, una peculiar conciencia de su precaria ubicación en el mundo a causa, en parte, de su condición de género; son débiles, feas, neuróticas y frustradas a causa de su incapacidad de vivir dignamente en un mundo desordenado donde, en la mayoría de los casos, tan sólo son sobrevivientes: "Used and abused, denied identity and fulfillment, Oates's women too often supinely accept the statement 'we are born only to be broken'. And break they do". 2

En su crítica de la "tecnología política del cuerpo" y de las prácticas disciplinarias coercitivas que buscan producir cuerpos dóciles, Foucault señala, en Vigilar y castigar, que el poder disciplinario se ejerce a través del juego de la mirada haciendo que los sometidos se sientan siempre observados, y usa el ejemplo del Panóptico para explicar cómo la visibilidad permanente controla tanto el cuerpo como la mente: "El que está sometido a un campo de visibilidad, y que lo sabe, reproduce por su cuenta las coacciones del poder; las hace jugar espontáneamente sobre sí mismo... se convierte en el principio de su propio sometimiento". ${ }^{3}$ Sin embargo, Foucault no habla de la manera en que dichas prácticas disciplinarias actúan sobre los cuerpos "dóciles" de las mujeres (históricamente sometidas al poder patriarcal y a los saberes que articulan y refuerzan dicho poder), ni de cómo en las sociedades contemporáneas la mirada fálica panóptica habita en la conciencia de la mayoría de las mujeres, naturalizando las diferencias producidas por el pensamiento binario así como las formas de sujeción que perpetúan el silencio y la impotencia. Así, si bien para este autor el alma es el elemento del cuerpo sobre el cual el poder se ejerce: "efecto e instrumento de una anatomía política; el alma, prisión del cuerpo", " parecería que en el caso de las mujeres la fórmula tendría que invertirse, haciendo del cuerpo femenino la prisión del ser.

Kathleen, la protagonista de The Rise of Life on Earth, es el ejemplo de una mujer que está siempre sometida a la mirada de los otros. Ha llegado a

${ }^{2}$ Mary Katherine GRANT, The Tragic Vision of Joyce Carol Oates, p. 28.

${ }^{3}$ Michel FoucaulT, Vigilar y castigar, p. 204.

4 Ibid., p. 36. 
este mundo, a este infierno material, como un ángel caído de un paraíso que en realidad nunca conoció. Después de haber sido golpeada por el padre (quien aparentemente mató a la hermana pequeña en esa misma ocasión) y abandonada por la madre, Kathleen se queda completamente sola y empieza su triste recorrido por diferentes casas para huérfanos de las que se "libera" con la mayoría de edad, hasta lograr realizar su sueño de ser enfermera. A pesar de su soledad, se las arregla para sobrevivir y hacer su vida en esta profesión de servicio (típicamente femenina) que le ayuda a acercarse a los otros, como se acercaron a ella las enfermeras cuando estuvo hospitalizada a los once años de edad, después del incidente con el padre. Ésta parece ser una de las múltiples maneras como "surge" la vida en esta tierra, parafraseando el título de la novela, en una realidad sórdida, hostil, casi trágica.

Pero el precario contacto que Kathleen establece con la humanidad resulta insuficiente, ya que no consigue nunca liberarse de la verdadera prisión, de ese microcosmos que es su propio ser. Sintiéndose siempre vigilada, Kathleen se sabe distinta no sólo por ser fea y debido a su desarrollo físico precoz (además de parecer un poco tonta), sino a causa de su orfandad y su abandono. Así, habiendo interiorizado su diferencia, se ve a sí misma como una freak, un ser grotesco sometido siempre a la mirada de los otros:

She'd overheard Mrs. Chesney on the telephone drawling to a friend, "-This one, this one I told you about, the sort of moony one, twelve years old but looks like, Jesus, maybe sixteen, not bad-looking except her skin's broken out, poor thing, they were trying to say she was retarded but it's just she's scared I think, Christ it's a hard life, I could tell you some things! - but this one she's sweet, she's sort of I guess strange- just needs love I guess, needs time to get, y'know, adjusted" (39).

Como otros personajes femeninos de Joyce Carol Oates, Kathleen está sola, no pertenece ni sabe cómo adaptarse al mundo exterior ya que está encerrada en su cuerpo de mujer, ese cuerpo marcado por la diferencia y que a los ojos de los demás resulta extraño. Es así como todos sus esfuerzos de adaptación fracasarán tarde o temprano a causa de la posición subordinada que ocupa dentro de la jerarquía de género que, en una sociedad patriarcal como la norteamericana, convierte a las mujeres, en términos generales, en seres marginales.

De acuerdo con Teresa de Lauretis, los sujetos se constituyen a partir de las representaciones y autorrepresentaciones culturales de género ( $\mathrm{y}$ no solamente por la diferenciación sexual), el cual es una elaboración cultural tan- 
to en su aspecto social como en su dimensión semiótica, es decir, como sistema de representaciones que otorgan sentido a la vida de los individuos en la sociedad. Siguiendo a Foucault, la autora señala que el género "como representación y autorrepresentación es también el producto de varias tecnologías sociales como el cine, y de discursos institucionales, epistemologías y prácticas críticas, así como de prácticas de la vida cotidiana".s Así, la experiencia de vida femenina es al mismo tiempo una experiencia de género, del proceso de creación de la propia subjetividad por parte de las mujeres una vez que han interiorizado las representaciones culturales y las posiciones sociales que les han sido asignadas y que las reducen a ser el Otro:

In other words, the threat of the feminine derives from the fact that it is defined by an act of marginalization, by a thrusting of "woman" to a position outside the order of the Same. Because she has been constructed not as self-same but as utterly different, she resists gestures of unification that belatedly attempt to control the implications of her Otherness. ${ }^{6}$

Desde esta perspectiva, resulta muy enriquecedor acercarnos a la obra de Joyce Carol Oates, muchos de cuyos personajes femeninos son individuos desadaptados que, además de pertenecer a las clases marginadas (aunque no siempre), se sitúan en relación de exterioridad con los discursos sociales hegémonicos por el hecho no tan simple de pertenecer al género femenino: se sienten aisladas en un mundo que es para ellas amenazante y que las mantiene al margen, no sólo por lo que son sino por lo que ellas mismas creen ser. Por una parte actúan como personas comunes y respetables, pero mantienen oculta esa parte "maldita" de su personalidad donde sé confunden sentimientos de inseguridad, de odio y de violencia reprimida:

Many of Oates's characters have this two-sided quality: one side ordinary and respectable, the other side ruled by such forbidden impulses as lust, violence, and hate. Often in her stories this under-side of the human personality erupts and compels these characters toward obsessive love-affairs, cruelty, brutality, and even murder. ${ }^{7}$

Muchas mujeres, al no considerarse hermosas de acuerdo con las normas de belleza de las sociedades occidentales, experimentan una especie de se-

Teresa de LAURETIS, Technologies of Gender, p. 2.

"Molly Hite, The Other Side of the Story, p. 159.

7 Joan D. WinsLow, "The Stranger Within: Two Stories by Oates and Hawthorne", en Elaine Showalter, ed., Where Are You Going, Where Have You Been, p. 91. 
paración entre el cuerpo y la mente, entre el yo y el súper yo, y la consecuencia inmediata es la desintegración de la identidad o bien una identidad escindida. Por ejemplo, cuando en sus oraciones Kathleen Hennessy reza por la gente que le es más cercana, se imagina que: "I stood before them, oh and I was not ugly or clumsy like a cow but seeing me they saw my soul like a shimmering flame they did not see me in the flesh at all but another standing where I stood: Praise God" (62).

Ella sabe que la mirada de los otros la convierte en alguien o en algo que no le gusta ser -aunque nunca llega a comprender quién es ella realmente- y preferiría ser otra, no ser vista como un cuerpo, como una vaca, como la "carne" que es. Para ella su cuerpo de mujer es deficiente, algo le falta pero también le sobra, es un cuerpo que le estorba, que la aprisiona y no le permite ser libre en un mundo donde las mujeres que no tienen belleza corporal parecerían no tener una identidad definida.

Estas mujeres se ven reflejadas en el espejo de una sociedad que las considera deficientes no solamente por pertenecer al género femenino (el mismo discurso psicoanalítico define a la mujer a partir de la envidia del pene que no se tiene o bien en términos de una carencia, de un hueco), sino por no cumplir con las expectativas de belleza necesarias para su aceptación social. Si resultan antifemeninas o grotescas, ello se debe a que la misma sociedad las juzga como anormales. Así, las mujeres feas o con algún defecto fisico deben hacer lo imposible por compensar esa "falta", pero su cuerpo difícilmente puede dejar de ser un obstáculo prácticamente insalvable para poder tener un lugar en el mundo. De esta manera, desde muy joven Kathleen se ve a sí misma como un ser monstruoso, fuera de lugar, en un mundo hostil y amenazante.

Al referirse al gótico femenino, la crítica Ellen Moers subraya la importancia que tienen en la literatura femenina norteamericana del siglo XX temas como el odio y el rechazo hacia una misma, así como la tendencia a la autodestrucción, y señala que la manera como las mujeres se ven a sí mismas es determinante en esta literatura:

Despair is hardly the exclusive province of any one sex or class in our age, but to give visual form to the fear of self, to hold anxiety up to the Gothic mirror of the imagination, may well be more common in the writings of women than of men. While I cannot prove this statistically, I can offer a reason: that nothing separates female experience from male experience more sharply, and more early in life, than the compulsion to visualize the self. ${ }^{8}$

${ }^{8}$ Ellen MOERs, Literary Women, p. 107. 
No obstante, lo que el espejo devuelve es la mirada de los otros, esa mirada que hace que un cuerpo sea considerado hermoso o monstruoso, que sea aceptado o rechazado. Así, la posición que ocupa el cuerpo grotesco, particularmente el femenino, es siempre de exterioridad, de marginalidad frente a lo "normal" que se define desde las convenciones sociales y culturales; es un cuerpo que aparece como incompleto y degradado, excesivo y al mismo tiempo en falta:

[...] the grotesque, particularly as a bodily category, emerges as a deviation from the norm. Normalization as it is enforced in what Teresa de Lauretis has referred to as the "technologies of gender" has been harsh and effective in its highly calibrated differentiation of female bodies in the service of a homogeneity called gender difference - that is, the (same) difference of woman from man. It might follow that the expression "female grotesque" threatens to become a tautology, since the female is always defined against the male norm. ${ }^{9}$

A diferencia de la concepción grotesca del cuerpo en la Edad Media y el Renacimiento que, según Bajtin, es producto de una visión orgánica del mundo donde "lo cósmico, lo social y lo corporal están ligados indisolublemente en una totalidad viviente e indivisible", ${ }^{10}$ en las sociedades contemporáneas el cuerpo grotesco conserva sólo sus aspectos negativos, encerrado en sí mismo y separado del mundo y de los otros. Con el desarrollo de las sociedades burguesas y el progresivo aislamiento de los individuos en sociedades donde ya no es posible celebrar la "fiesta utópica" carnavalesca, en la cual todos son uno y uno es todos, el grotesco se vuelve hacia adentro y se convierte en "un grotesco de cámara, una especie de carnaval que el individuo representa en soledad, con la conciencia agudizada de su aislamiento"," señala Bajtin en relación con el grotesco romántico. El cuerpo grotesco es ahora un cuerpo que ya no goza sino que sufre su diferencia, su "anormalidad", un cuerpo paralizado por su impotencia y por su otredad, que ha dejado de reconocerse como miembro de un colectivo coherente y organizado.

En el mundo moderno y en una sociedad como la norteamericana, donde la comunidad ha dejado de funcionar como tal y probablemente ha dejado de ser viable, los individuos se encuentran particularmente aislados. Mary Katherine Grant opina que en la obra de Joyce Carol Oates: "Nowhere are

\footnotetext{
${ }^{9}$ Mary Russo, The Female Grotesque, p. 11.

${ }^{10}$ Mijail BAJTIN, La cultura popular en la Edad Media y Renacimiento, p. 23.

1 Ibid., p. 40.
} 
there cohesive bonds - not in family relationships, in the work community, in the neighborhood, or the gang, in male-female ties- in the whole of her fiction, there is no enduring bond among human beings". ${ }^{12}$

En el caso de la familia, el núcleo comunitario original, la ruptura suele generarse desde la infancia o en la adolescencia tal como sucede en muchas novelas del gótico femenino (como las de Flannery O'Connor, Carson McCullers y la misma Joyce Carol Oates). Asi, en The Rise of Life on Earth el acto de violencia del padre con el que da inicio la novela es en parte el origen de lo que va a sucederle a Kathleen. El padre agrede físicamente a sus dos hijas, pero ella misma, en su desesperación por hacer callar a su hermanita, la golpea hasta provocarle la muerte.

De esta manera surge a la vida este "monstruo" que a la larga va a cobrarse la violencia con que ha sido engendrada (en-generada), violencia que va a repetirse en el mundo exterior, particularmente en sus relaciones con los hombres. Todos los intentos de este personaje por conseguir el amor de una familia o de un hombre, o incluso de sus compañeras de trabajo, resultan infructuosos: el amor parece imposible para una "freak" como Kathleen y lo resiente en carne propia:

When they laughed at Kathleen Hennessy their laughter was sometimes like tickling, Kathleen writhed with the delight of it, like an infant's her entire body was suffused with pleasure, but at other times their laughter was jeering, jabbing, like hard malicious fingers in her ribs or between her legs or one of the older boys at school pinching her breasts, the very nipples twisted and scorned (36).

Sin embargo, frente a este rechazo, frente a esta violencia ejercida en contra de su cuerpo, Kathleen elabora una estrategia de resistencia: "Power resided within her, though. A match lighted in a strategic place, at night..." (39). Y así como descubrimos que ella fue quien mató "accidentalmente" a su hermana pequeña, sospechamos también que provocó el incendio en la casa de Mrs. Chesney de manera premeditada, a manera de venganza, y más adelante la vemos convertirse en la causante de varias muertes por contagio de hepatitis en el hospital donde trabaja. Sin embargo, sus venganzas no son más que una muestra más de su impotencia, ya que siempre afectan a los más débiles, incluyendo a ella misma. Los constantes esfuerzos de comunicación y de adaptación a lo largo de su vida han sido inútiles puesto que no puede evitar ser como es, un rostro y un cuerpo desagradables para

12 M. K. GRANT, op. cit., p. 25. 
los demás, y el rechazo definitivo a su persona se deberá no sólo a su fealdad sino a algo que va a sucederle a su cuerpo.

Kathleen mantiene una relación sexual (que no amorosa) con Orson Abbott, un médico perteneciente a una clase social más alta y que la utiliza exclusivamente como un objeto para su propia satisfacción física. Narrados desde el punto de vista de Orson, los momentos de encuentro sexual resultan particularmente grotescos ya que, para él, esta mujer desconocida hacia quien no siente ningún afecto es sólo un cuerpo, dócil y manipulable, siempre disponible:

[...] he was murmuring words he could not himself quite hear, Love love love oh God, oh God in a delirium of desire as she winced at his embrace yet unprotesting acquiesced to it, always she whoever she was, this one, this short dumpy big-breasted one, sweet-faced one, baby cow, silky cunt, always she acquiesced... (108-109).

Al descubrir que está embarazada, Kathleen se da cuenta también de que si bien es tolerada como un cuerpo para el placer del otro, obviamente no puede pretender ser más que eso, y su embarazo va a provocar un alejamiento definitivo por parte de su amante. Ella había deseado tener un bebé desde que vivía en la casa de huérfanos de Mrs. Chesney, y al ver a uno había fantaseado que "He'd pushed out from between her legs from that secret place up inside her (she'd seen drawings of it, diagrams in the Collier's Encyclopedia at the public library) meaning he was hers" (43). Abandonada a su suerte y a manera de venganza frente a Abbott o frente al mundo, decidirá provocarse ella misma un aborto echando mano de los conocimientos de medicina adquiridos en su trabajo hospitalario. No obstante, esta decisión no afectará a nadie más que a la misma Kathleen, quien va a ejercer un terrible acto de violencia, una especie de autocastigo contra su propio cuerpo que parece haberse convertido en su principal enemigo: "In Oates's world, violence is performed by the character himself, motivated by his own sense of powerlessness. It does not radically change his life, although it may be a means of his temporarily transcending his petty existence"..$^{13}$

Así, a partir de este momento decisivo en su vida, Kathleen parece haber adquirido cierto poder: el poder de decidir sobre la vida y la muerte, $y$ con ello, quizá, una nueva identidad. Aunque en realidad este poder es antes que nada autodestructivo y va a dejarla más sola que nunca, sin amigos, sin amante, sin el bebé que ha tenido que sacrificar y sin ninguna esperanza de que su vida cambie:

${ }^{13}$ Ibid., p. 35. 
[...] so she lay very still understanding how in the human world she was invisible and would forever be thus and so her pain and her suffering if it was suffering and the death of the tiny skinless thing meant to be her baby if it was a true death did not matter in the human world in the slightest You're shit: pig's snout and seemed not to matter much in God's world and she wondered if there were others of her kind and if these others knew of one another and knew of one another's secret strength, the terrible secret strength of those whom the human world has made invisible... (132).

El mundo de Kathleen parece haberse convertido en un lugar aterrador donde los que como ella son "invisibles" parecen quedar aislados de manera definitiva. Sin embargo, paradójicamente, esa misma invisibilidad va a convertirse en su fortaleza en la medida en que representa una forma de resistencia frente al poder patriarcal - una suerte de escape de los códigos genéricos culturales - y la única posibilidad de que Kathleen pueda conformar una identidad propia al identificarse con otros, aunque sea desde esta dudosa posición.

Resulta irónico que este personaje se haga fuerte a partir de su extrema debilidad, que "surja" a la vida al provocar la muerte y que su acto destructivo no provoque, como en el caso del protagonista trágico, una caída definitiva sino que simplemente sea un acontecimiento más entre tantos otros en la vida de esta mujer solitaria. Después de haberse sentido constantemente observada por los demás, después de haber estado siempre sometida a la mirada de los otros, Kathleen descubre finalmente que en realidad nunca ha sido vista: "Oh why not? why not me - Kathleen'? Why do none of you see me? and most of the time their eyes went through her not cruel or scornful simply not seeing..." (79), y que ese cuerpo tan lleno de vida para ella en realidad ha pasado inadvertido y ha sido siempre, para los otros, prácticamente inexistente. No obstante, para Kathleen dicha invisibilidad se convertirá por una parte en una forma de liberación y por otra en una increíble fuente de poder destructor, ya que no tendrá que responder de sus actos ni ante los hombres ni ante Dios. A diferencia del ejemplar héroe trágico, ha comprendido que es una víctima anónima y que en su terrible soledad queda totalmente "excluded from the human world of power and authority and responsibility" (134).

A partir de este momento en la vida de Kathleen ya nada parece tener sentido, al menos no un sentido trascendente, ya que al quedar al margen de la sociedad queda también al margen de cualquier sistema absoluto de valores. Para Jan Kott: "In the world of the grotesque, downfall cannot be justified by, or blamed on, the absolute. The absolute is not endowed with any ultimate 
reasons; it is stronger, and that is all. The absolute is absurd". ${ }^{14}$ Éste parece ser el verdadero aprendizaje de este personaje, de esta mujer que vive una vida oculta, prisionera en su propio cuerpo, y que, en su esfuerzo por desconstruir el poder de la mirada del otro, vuelve a nacer, a "surgir" a la vida en esta tierra, esta vez con la terrible conciencia de su soledad y de su exclusión del mundo de los otros pero también del poder que su invisibilidad le otorga.

\section{Bibliografia}

BAJTIN, Mijail, La cultura popular en la Edad Media y Renacimiento. Barcelona, Barral, 1974.

FOUCAULT, Michel, Vigilar y castigar. México, Siglo XXI, 1996.

GRANT, Mary Katherine, The Tragic Vision of Joyce Carol Oates. Durham, N. C., Universidad Duke, 1978.

HITE, Molly, The Other Side of the Story. Nueva York, Universidad Cornell, 1989.

KOTT, Jan, Shakespeare Our Contemporary. Londres, Routledge, 1991.

LAURETIS, Teresa de, Technologies of Gender. Bloomington, Universidad de Indiana, 1987.

MOERs, Ellen, Literary Women. Nueva York, Universidad de Oxford, 1985.

OATES, Joyce Carol, The Rise of Life on Earth. Nueva York, New Directions, 1991.

OATES, Joyce Carol, Wonderland. Nueva York, Ontario Review Press, 1992.

Russo, Mary, The Female Grotesque, Nueva York, Routledge, 1994.

WINSLOW, Joan D., "The Stranger Within: Two Stories by Oates and Hawthorne", en Elaine SHOWALTER, ed., Where are You Going, Where Have You Been. Nueva Jersey, Universidad de Rutgers, 1994.

14 Jan KoTT, Shakespeare Our Contemporary, p. 105. 